Received: 2019.02 .03

Accepted: 2019.03.29

Published: 2019.04 .24

\title{
The Role of Regulatory B Cells in Patients with Acute Myeloid Leukemia
}

Authors' Contribution: Study Design A Data Collection B Statistical Analysis C Data Interpretation D Manuscript Preparation E Literature Search F Funds Collection $G$

Corresponding Author: Source of support:

Background:

Material/Methods:

Conclusions:

MeSH Keywords:

Full-text PDF:
Zhuogang Liu, e-mail: liuzg@sj-hospital.org

This work was supported by The Natural Science Foundation of China (No. 81600115)
China Medical University, Shenyang, Liaoning, P.R. China

AG Zhuogang Liu

Results: We found that the frequency of Breg cells was higher in AML patients both in peripheral blood (PB) and in bone marrow (BM) compared with those in HDs. The AML patients with high WBC levels had higher Breg cell frequency compared with those with low WBC levels. Low-risk patients with had lower Breg cells frequency compared to the medium-risk patients. The patients with high WBC and high Breg cells frequency showed a shorter overall survival. Similarly, the overall survival of AML patients in the older group with high Breg cells frequency was significantly shorter than in the younger group with low Breg cell frequency.

For AML patients, the frequency of Breg cells was elevated, and high frequency of Breg cells may reveal poor prognosis.

B-Lymphocytes, Regulatory • Leukemia, Myeloid, Acute • Prognosis

https://www.medscimonit.com/abstract/index/idArt/915556

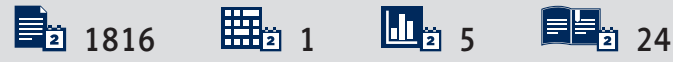




\section{Background}

Regulatory B (Breg) cells are a group of B cells with immunomodulatory function, which mainly exert negative immunomodulatory function by secreting IL-10 and other cytokines. IL-10-secreting Breg cells are involved in the pathogenesis of systemic lupus erythematosus (SLE), rheumatoid arthritis (RA), and multiple sclerosis (MS) and play an important role in preventing the occurrence of autoimmune diseases [1]. Studies have also reported that Breg cells could improve graft-versushost disease (GVHD) by secreting IL-10 or TGF- $\beta[2,3]$. Breg cells were also found to be involved in the pathological process of oesophageal carcinoma, gastric cancer, and human hepatocellular carcinoma [4-6]. Breg cells can inhibit the immune response of effector $\mathrm{T}$ cells to breast cancer, prostate cancer, and skin cancer and may participate in the immune regulation of tumors $[7,8]$.

Acute myeloid leukemia (AML) is a hematological malignancy with excessive proliferation of immature myeloid cells, which results in impaired differentiation and maturation. High white blood cell (WBC) index is a prognostic factor for AML [9]. High percentages of $C D 19+B$ cells in newly diagnosed AML patients were reported to be unrelated to DFS and OS [10]. However, it has been reported that the transitional $B$ cell frequency of patients with AML was higher than that of healthy controls, and patients sampled further from the end of chemotherapy had lower transitional B cell frequency [11]. The pathogenesis of AML is not yet fully elucidated and it is well recognized that abnormalities in the immune system are involved in the pathogenesis of AML. It is generally believed that immune cells affecting cancer mainly include regulatory $T$ cells, effector $T$ cells, and $B$ cells. Due to their immunoregulatory properties, Breg cells participate in the pathogenesis of AML. Nevertheless, the expression profile of Breg cells in AML has so far been poorly explored. The purpose of our study was to assess the frequency of Breg cells and the relationship between the Breg cells and clinical data of patients with AML.

\section{Material and Methods}

\section{Patients and controls}

A total of 46 cases newly diagnosed AML patients at Sheng Jing Hospital of China Medical University from November 2017 through February 2018 were enrolled in our study. We excluded acute promyelocytic leukemia patients. Diagnoses were carried out on the basis of the French-American-British (FAB) classification system.

We enrolled 15 healthy donors (HD) as healthy controls. Samples were obtained from the healthy donors only if they were not in abnormal immune status. Our study was authorized by the local Ethics Committee.

\section{Procedures of specimen collection and conduct}

Peripheral blood mononuclear cells (PBMC) and bone marrow mononuclear cells (BMMC) were separated with lymphocyte separation reagents by density gradient centrifugation according to the manufacturer's recommendations.

Specific human anti-CD38- fluorescein isothiocyanate (cat. no. A07778), anti-CD24-phycoerythrin (cat. no. IM1428U), and anti-CD19- phycoerythrinCy-5 (cat. no. A07771) monoclonal antibodies were used to identify the phenotypes of cells. Antibodies were obtained from BECKMAN COULTER (Brea, CA).

For surface staining, cells from PBMC or BMMC were stained for 30 min at $4^{\circ}$ away from light with CD19- phycoerythrinCy-5, CD24phycoerythrin, and CD38- fluorescein isothiocyanate for extracellular markers of Breg cells. After staining, cells were washed once in normal saline and resuspended in $340 \mathrm{ul} \mathrm{normal} \mathrm{saline,} \mathrm{and} \mathrm{the}$ lymphocytes were gated according to FSC/SSC criteria. To confirm the specificity of the monoclonal antibody, homotypic controls were set for each fluorochrome. The results for Breg cells analysis are expressed as percentages of total CD19+ B lymphocytes.

For immunophenotypic analysis, we used Kaluza Analysis Software version 2.0. (BECKMAN COULTER). Breg cells were described as CD19+CD24+CD38+. For each sample, a minimum of $2 \times 10^{5}$ events in the gate of live cells were computed.

\section{Chemotherapy regimens}

Patients were treated with induction chemotherapy (7 days of continuous infusion cytarabine with 3 days of anthracycline) and consolidation therapy (medium/high-dose cytarabine based chemotherapy) and no patients underwent allogeneic transplantation during our research.

\section{Statistical analysis}

Quantitative data are expressed as the mean plus or minus the standard. Differences in the frequency of Breg cells were assessed using Wilcoxon's test or Mann-Whitney $U$ test. Correlations between Breg cells and clinical data were assessed by Spearman's correlation test. We considered all tests to be double-tailed with an $\alpha$ level of 0.05 , which was statistically significant. Analyses were carried out using the GraphPad PRISM 5.0 software. Kaplan-Meier method was used for survival analysis. We used the log-rank and Breslow-Gehan-Wilcoxon tests to compare survival curves. The initial time for survival was the time of blood collection. All patients who survived were reviewed at the time of last follow-up for survival analysis. 
Table 1. Demographic characteristics of patients with AML and HD.

\begin{tabular}{|c|c|c|c|c|c|}
\hline \multirow{3}{*}{\multicolumn{2}{|c|}{ Characteristic }} & \multicolumn{3}{|c|}{ Finding } & \multirow{3}{*}{$P$ value } \\
\hline & & \multicolumn{2}{|c|}{ AML $(n=46)$} & \multirow[b]{2}{*}{ HD $(n=15)$} & \\
\hline & & PB $(n=36)$ & BM $(n=10)$ & & \\
\hline Age (mean $\pm \mathrm{S}$ & & $49.64 \pm 15.02$ & $42.60 \pm 23.50$ & $46.67 \pm 17.01$ & 0.7309 \\
\hline Female, No. (\% & & $63.9 \%$ & $50.0 \%$ & $73.3 \%$ & 0.7455 \\
\hline \multirow{4}{*}{ FAB subtypes } & M1 No. & 2 & 1 & & \\
\hline & M2 No. & 11 & 4 & & \\
\hline & M4 No. & 8 & 2 & & \\
\hline & M5 No. & 15 & 3 & & \\
\hline
\end{tabular}

\section{Results}

The demographic characteristics of the patients with $A M L$ and the HD included in the study are given in Table 1. A total of 46 patients and 15 healthy donors (as a control group) were included in the study.

The mean $\pm S D$ age of these patients with Bregs in peripheral blood (PB) was $49.64 \pm 15.02$ years, and 23 (63.9\%) patients were women. The mean \pm SD age of these patients with Bregs in bone marrow (BM) was $42.60 \pm 23.50$ years, and $5(50.0 \%)$ patients were women. The mean \pm SD age of HDs was $46.67 \pm 17.01$ years, and 11 (73.3\%) healthy donors were women. There was no statistically significant difference between the patient group and the HD group in terms of demographic findings.

\section{Frequency of Breg in AML patients and HDs}

As exhibited in Figure 1, the frequency of Bregs was higher in AML patients, both in $\mathrm{PB}$ and in BM, compared with that in HDs. Nevertheless, no differences between the PB and BM were discovered. A mean value of Breg cells of $25.29 \pm 10.68 \%$ (range, $4.980-44.70 \%$ ) in PB and $26.74 \pm 15.76 \%$ (range, $12.50-64.20$ ) in $\mathrm{BM}$ was observed in patients compared with $11.16 \pm 9.601 \%$ (range, $1.050-38.20 \%)$ in HDs $(P<0.0001, P=0.0242$, respectively). In summary, our data suggest that the AML patients have a significant rise in the frequency of Breg cells when compared to controls.

\section{Association between Breg cells and clinical data}

As shown in the Figure 2, there was no statistically significant difference in FAB subtypes of leukemia (Figure 2A). The AML patients were divided into 2 groups by the median of WBC. The AML patients with high WBC had higher frequency of Breg cells compared with the low WBC group $(P=0.0251)$ (Figure $2 B$ ). The frequency of Breg cells was comparable between the older and younger groups $(\mathrm{P}=0.6622)$ (Figure $2 \mathrm{C}$ ).

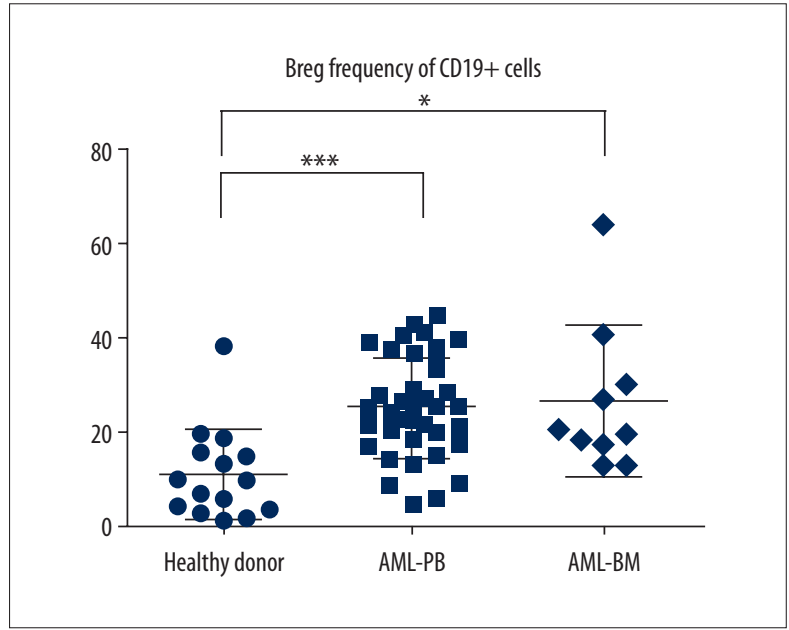

Figure 1. Expression of Breg cells between $A M L$ and healthy donors. $\mathrm{PB}$ - peripheral blood; BM - bone marrow; NS - no significance. * Means $\mathrm{P}<0.05 ;{ }^{* * *}$ means $P<0.0001$.

The patients with good prognosis had a decreasing trend of Breg cells frequency compared to the medium prognosis group, poor prognosis group, and unknown prognosis group, but no significant differences were found (Figure $3 \mathrm{~A}$ ). The low-risk patients had lower Breg cells frequency compared to the medium-risk patients $(P=0.0097)$ (Figure 3B).

To investigate whether Breg cells affect clinical outcome, the survival of AML patients was examined. There were no significant differences between the high WBC group and the low- WBC group $(P=0.3778)$ (Figure $4 A)$. When the high WBC group was further classified into 2 groups using the median of Breg cells positivity, cases in the high-Breg group showed a shorter overall survival compared with the low-Breg group $(P=0.0119)$ (Figure 4B). We also assessed the influence of age and Breg cells frequency on patient survival. There was similar overall survival between the younger group and the older group (Figure 5A). Next, the patients were divided into 4 groups based on the median frequency of Breg cells and the 

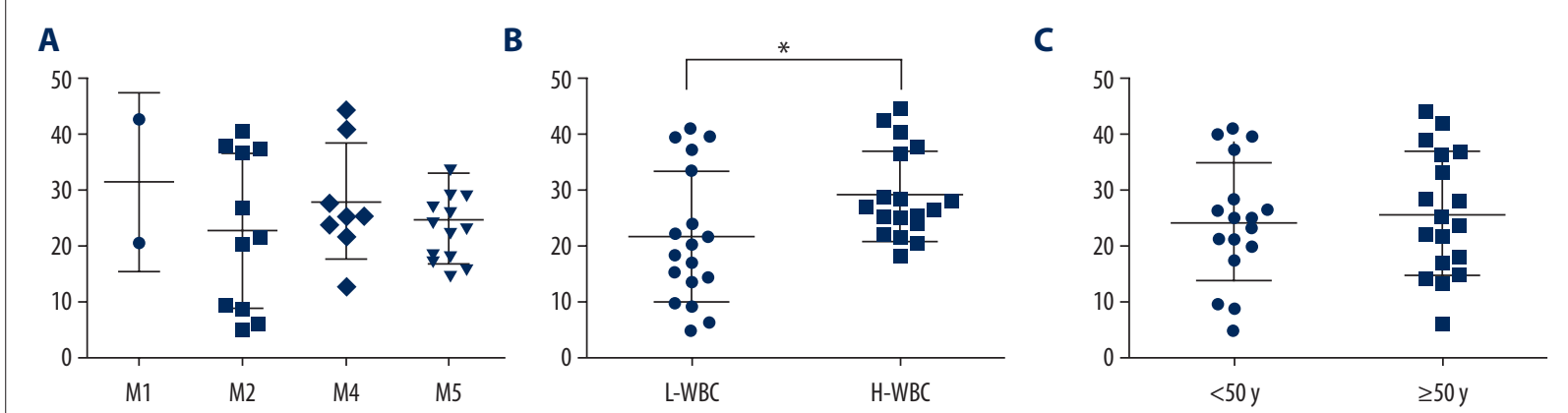

Figure 2. Breg frequency of $A M L$ in PB. (A) Breg frequency in different $F A B$ subtypes of leukemia; (B) Breg frequency with different WBC levels; (C) Breg frequency with different age groups; PB - peripheral blood. * Means $P<0.05$.

A

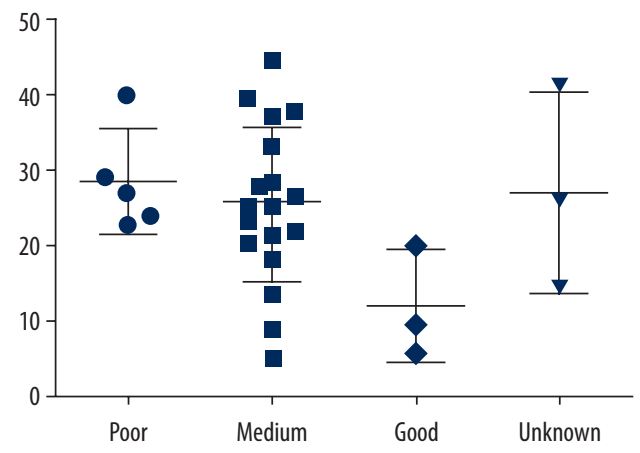

B

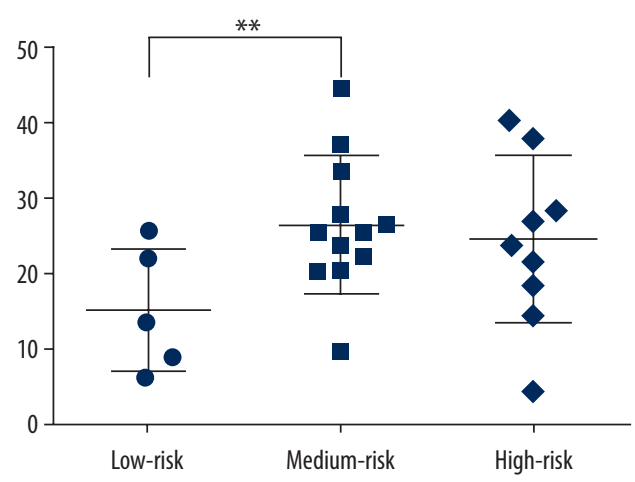

Figure 3. Breg frequency of AML in PB. PB - peripheral blood. (A) The frequency of Bregs in different SWOG groups of leukemia. (B) The frequency of Bregs with different prognosis. ${ }^{* *}$ Means $\mathrm{P}<0.01$.

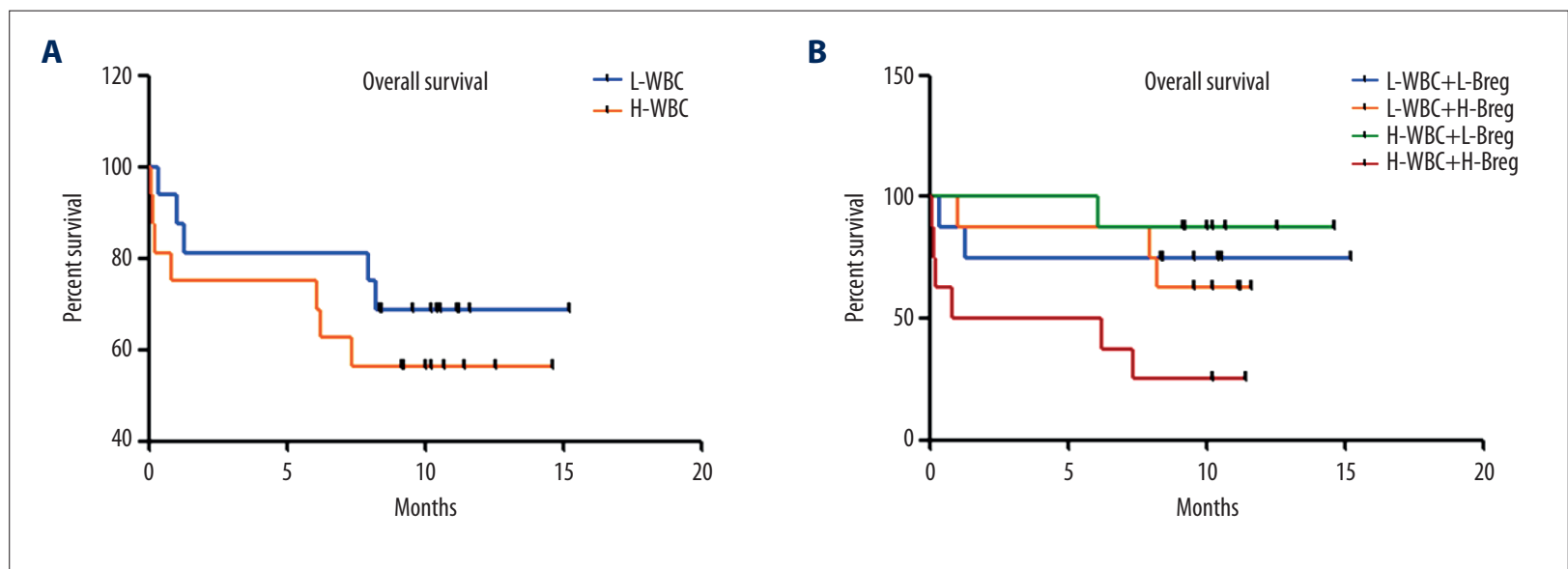

Figure 4. Overall survival of AML. (A) The overall survival in different WBC groups. (B) The overall survival in different WBC and Breg groups.

median age. The overall survival in the older group with high Breg frequencies were significantly shorter than in the younger group with low Breg cell frequency ( $P=0.0272$ ) (Figure 5B), suggesting that increased Breg cell frequency reflects poor prognosis in AML patients.

\section{Discussion}

Recent studies have found that there is a group of B cells with immunomodulatory function, which mainly exert negative immunomodulatory function by secreting IL-10 and 

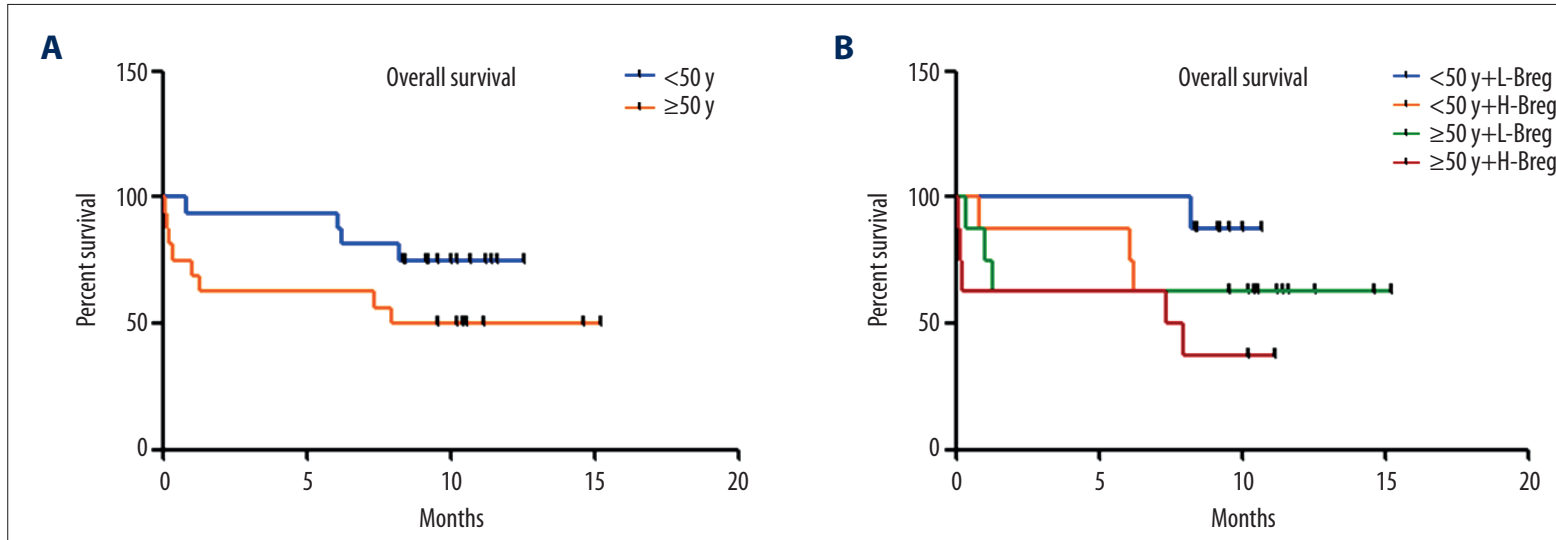

Figure 5. Overall survival of AML. (A) The overall survival in different age groups. (B) The overall survival in different age and Breg groups.

other cytokines. Studies have found that in autoimmune diseases such as SLE, RA, MS, and myasthenia gravis (MG), Breg cells frequency was decreased $[1,12,17]$ indicating that IL-10secreting Breg cells are involved in the pathogenesis of autoimmune diseases and play an important role in preventing autoimmune diseases. Chang et al. [2] found that donor-derived CD19+CD24highCD38high Breg cells inhibit CD4+T cells by secreting TGF- $\beta$ and IL-10, thereby regulating acute GVHD after hematopoietic stem cell transplantation (HSCT). Peng et al. [3] found mesenchymal stem cells (MSC) infusion upregulated Breg cells, which consequently improved chronic GVHD by secreting IL-10. Breg cell also play an important role in the course of tolerance induction and maintenance of kidney transplantation and islet allotransplantation [18,19]. The level of Breg cells of patients with oesophageal carcinoma and gastric cancer was significantly higher than that in healthy controls $[4,5]$. Boulassel et al. [20] found the levels of Breg cells were lower in patients with $\mathrm{B}$ cell non-Hodgkin lymphoma ( $\mathrm{NHL}$ ) compared with that of healthy controls, and the frequency of Breg cells was remarkably increased after R-CHOP chemotherapy. Previous research has shown that Breg cells promote human hepatocellular carcinoma (HCC) growth and invasiveness [6].

Li et al. [21] found that a decrease in the percentage of Breg cells indicated a better prognosis in patients with advanced gastric cancer. Shao et al. [6] revealed that human Breg cells promoted human hepatocellular carcinoma tumor growth in severe combined immune deficiency (SCID) mice. Song et al. [22] reported that an increased number of IL-10-producing Breg cells might play important roles in human hepatocellular carcinoma progression. In our study, the frequency of Breg cells markedly increased in patients with AML compared with HDs. We also found that the patients with high WBC and high Breg cells frequency showed a shorter overall survival, and the overall survival of AML patients in the older group with high Breg frequency was significantly shorter than those in the younger group with low Breg cells frequency. Previous studies have reported the elevated WBC was associated with shorter survival $[9,23,24]$. The raised WBC might include abnormal granulocytes derived from leukemic precursors, which result in a poor prognosis. On the other hand, we found that the AML patients with high WBC levels had higher Breg cells frequency. Our results suggested that immune factors were involved in the prognosis of AML. We also found that patients with low risk or good prognosis had lower Breg cells frequency. From this point of view, Breg cells can be used to screen high-risk AML patients and provide a theoretical basis for future immunotherapy.

Our research has some limitations. The study had a relatively small sample size. Moreover, because the sample availability was limited, we did not perform functional cellular assays. Further studies on the specific functions of Breg cells are warranted.

\section{Conclusions}

In conclusion, our findings suggest that AML patients had higher frequency of Breg cells, which predicted a shorter overall survival and poor prognosis, suggesting that Breg cells are involved in AML pathogenesis. All these results open a new avenue in the study of tumor immunotherapy. Further studies are needed to clarify their specific roles in the pathophysiology of AML occurrence and development, finally providing advances in clinical treatment.

\section{Conflict of interest}

None. 


\section{References:}

1. Miyagaki T: [Regulatory B cells in human autoimmune diseases]. Nihon Rinsho Meneki Gakkai Kaishi, 2015; 38(5): 390-97 [in Japanese]

2. Chang Y-J, Zhao X-S, Lv M et al: CD19+CD24highCD38high B cells exhibit higher ability in TGF-\{beta\} and IL-10 secretion and suppressing of CD4+ T cell proliferation but are quantitatively decreased in G-BM of healthy donors (P2146). J Immunol, 2013; 190(1 Suppl.): 69.11

3. Peng $Y$, Chen $X$, Liu $Q$ et al: Mesenchymal stromal cells infusions improve refractory chronic graft versus host disease through an increase of CD5+ regulatory B cells producing interleukin 10. Leukemia, 2015; 29(3): 636-46

4. Qian L, Bian GR, Zhou Y et al: Clinical significance of regulatory B cells in the peripheral blood of patients with oesophageal cancer. Cent Eur J Immunol, 2015; 40(2): 263-65

5. Li G, Wulan H, Song $Z$ et al: Regulatory $B$ cell function is suppressed by smoking and obesity in $\mathrm{H}$. pylori-infected subjects and is correlated with elevated risk of gastric cancer. PLoS One, 2015; 10(7): e0134591

6. Shao Y, Lo CM, Ling CC et al: Regulatory B cells accelerate hepatocellular carcinoma progression via CD40/CD154 signaling pathway. Cancer Lett, 2014; 355(2): 264-72

7. He Y, Qian H, Liu Y et al: The roles of regulatory B cells in cancer. J Immunol Res, 2014; 2014: 215471

8. Gorosito Serran M, Fiocca Vernengo F, Beccaria CG et al: The regulatory role of $B$ cells in autoimmunity, infections and cancer: Perspectives beyond IL10 production. FEBS Lett, 2015; 589(22): 3362-69

9. Nguyen $S$, Leblanc T, Fenaux $P$ et al: A white blood cell index as the main prognostic factor in $\mathrm{t}(8 ; 21)$ acute myeloid leukemia (AML): A survey of 161 cases from the French AML Intergroup. Blood, 2002; 99(10): 3517-23

10. Park Y, Lim J, Kim S et al: The prognostic impact of lymphocyte subsets in newly diagnosed acute myeloid leukemia. Blood Res, 2018; 53(3): 198-204

11. Goswami M, Prince G, Biancotto A et al: Impaired B cell immunity in acute myeloid leukemia patients after chemotherapy. J Transl Med, 2017; 15(1): 155

12. Yilmaz V, Maillard S, Truffault $F$ et al: Regulatory B cells in myasthenia gravis are differentially affected by therapies. Ann Clin Transl Neurol, 2018; 5(11): 1408-14

13. Santaguida MG, Gatto I, Mangino G et al: Breg cells in celiac disease isolated or associated to Hashimoto's thyroiditis. Int J Endocrinol, 2018; 2018 5290865
14. von Borstel A, Lintermans LL, Heeringa P et al: Circulating CD24hiCD38hi regulatory $B$ cells correlate inversely with the ThEM17 cell frequency in granulomatosis with polyangiitis patients. Rheumatology (Oxford), 2019 [Epub ahead of print]

15. Watanabe R, Ishiura N, Nakashima $\mathrm{H}$ et al: Regulatory B cells (B10 cells) have a suppressive role in murine lupus: CD19 and B10 cell deficiency exacerbates systemic autoimmunity. J Immunol, 2010; 184(9): 4801-9

16. Blair PA, Norena LY, Flores-Borja F et al: CD19(+)CD24(hi)CD38(hi) B cells exhibit regulatory capacity in healthy individuals but are functionally impaired in systemic Lupus Erythematosus patients. Immunity, 2010; 32(1): $129-40$

17. Flores-Borja F, Bosma A, Ng D et al: CD19+CD24hiCD38hi B cells maintain regulatory $\mathrm{T}$ cells while limiting $\mathrm{TH} 1$ and $\mathrm{TH} 17$ differentiation. Sci Trans Med, 2013; 5(173): 173ra23

18. Latorre I, Esteve-Sole A, Redondo D et al: Calcineurin and mTOR inhibitors have opposing effects on regulatory $T$ cells while reducing regulatory $B$ cell populations in kidney transplant recipients. Transpl Immunol, 2016; 35: 1-6

19. Li S, Li X, Yang M et al: Identification of the subsets of IL-10-producing reg ulatory $B$ cells in the course of tolerance induction and maintenance in islet allotransplantation. Transplant Proc, 2018; 50(10): 3900-5

20. Boulassel MR, Al Qarni Z, Burney I et al: Levels of regulatory T cells and invariant natural killer cells and their associations with regulatory $B$ cells in patients with non-Hodgkin lymphoma. Mol Clin Oncol, 2018; 9(6): 677-82

21. Li W, Song D, Li H et al: Reduction in peripheral CD19+CD24hCD27+ B cell frequency predicts favourable clinical course in XELOX-treated patients with advanced gastric cancer. Cell Physiol Biochem, 2017; 41(5): 2045-52

22. Song S, Yuan P, Li P et al: Dynamic analysis of tumor-associated immune cells in DEN-induced rat hepatocellular carcinoma. Int Immunopharmacol, 2014; 22(2): 392-99

23. Billstrom R, Johansson $B$, Fioretos T et al: Poor survival in $t(8 ; 21)$ ( $q 22 ; q 22)$ associated acute myeloid leukaemia with leukocytosis. Eur J Haematol, 1997; 59(1): 47-52

24. O'Brien S, Kantarjian HM, Keating M et al: Association of granulocytosis with poor prognosis in patients with acute myelogenous leukemia and translocation of chromosomes 8 and 21. J Clin Oncol, 1989; 7(8): 1081-86 